\title{
The low-frequency radiotelescope NenuFAR
}

Philippe Zarka ${ }^{(1,2)}$, Andrée Coffre ${ }^{(2)}$, Laurent Denis ${ }^{(2)}$, Cédric Dumez-Viou ${ }^{(2)}$, Julien Girard ${ }^{(3,1)}$, Jean-Mathias Grießmeier ${ }^{(4,2)}$, Alan Loh ${ }^{(1,2)}$, Michel Tagger ${ }^{(4,2)}$, and the NenuFAR-France consortium

(1) LESIA, Observatoire de Paris, CNRS, PSL, Meudon, France, e-mail: philippe.zarka@obspm.fr; alan.loh@obspm.fr

(2) Station de Radioastronomie de Nançay, Observatoire de Paris, CNRS, PSL, Université d'Orléans, Nançay, France, e-mail: andree.coffre@obs-nancay.fr; laurent.denis@obs-nancay.fr; cedric.viou@obs-nancay.fr

(3) CEA/AIM, Université Paris Diderot, Saclay, France, e-mail: julien.girard@cea.fr

(3) LPC2E, CNRS, Université d’Orléans, Orléans, France, e-mail: jean-mathias.griessmeier@enrs-orleans.fr; michel.tagger@cnrs-orleans.fr

NenuFAR (New extension in Nançay upgrading LOFAR), is a low-frequency SKA pathfinder presently in construction and commissioning in Nançay (France) [1,2]. NenuFAR consists of 102 mini-arrays (MA) of 19 dual polarization antennas each. $96 \mathrm{MA}$ cover densely a disk (core) of $400 \mathrm{~m}$ in diameter, and $6 \mathrm{MA}$ are located at distances up to $\sim 3 \mathrm{~km}$. NenuFAR will cover the range 10-85 MHz, that encompasses the low-frequency range of LOFAR ([3] 0-80 MHz nominal, that can be extended to 10-85 MHz), and be three instruments in one:

(i) the core, connected to the receivers of the French LOFAR (FR606) station, will form a giant LOFAR station that can replace the LOFAR FR606 LBA field and be used as part of LOFAR; this LOFAR Super Station (LSS) will improve the sensitivity of long baselines by over one order of magnitude and thus the quality of high angular resolution (sub-arcsecond) imaging at low frequencies with LOFAR.

(ii) the core will simultaneously be a standalone compact beamformer providing high instantaneous sensitivity, that will allows us to search efficiently for weak unresolved sources such as pulsars, exoplanets, stars ... A dedicated pulsar/time/frequency backend is in development, and the possibility to connect a SETI receiver that will analyze all NenuFAR data in piggyback (commensal) mode is under study.

(iii) finally, thanks to the addition of a GPU-based correlator, NenuFAR will also (and simultaneously) be a standalone imager that can be used in two modes: a fast $(1 \mathrm{sec})$ coarse resolution $\left(1^{\circ}\right)$ mode, using the $96 \mathrm{MA}$ of the core only, and a slow (6-8 hours) medium resolution ( $\left(8^{\prime}\right)$ mode using all the $102 \mathrm{MA}$, core + distant; this mode will enable imaging studies in cosmology (search for the dark ages / cosmic dawn signal), planetary and stellar physics (exoplanets, stars and their plasma interactions), and the study of galaxies, clusters and haloes.

The operation of this several-in-one instrument will be complex, and will take advantage of original developments including its graphic control/command interface and the management of polluted frequencies bands. Ongoing commissioning includes end-to-end instrument simulations that helped to understand and overcome various difficulties. The science case of NenuFAR [3] has been developed with inputs from a consortium (NenuFAR-France, that includes non-French participants), which is open to broader participation, and in the process of structuring itself in Key Scientific Projects. Early science observations are expected before the end of this year (2018).

1. P. Zarka, J. N. Girard, M. Tagger, L. Denis, and the LSS team, "LSS/NenuFAR: The LOFAR Super Station project in Nançay", SF2A-2012: Proc. Annual meeting of the French Society of Astronomy and Astrophysics, Eds.: S. Boissier, P. de Laverny, N. Nardetto, R. Samadi, D. Valls-Gabaud and H. Wozniak, pp.687-694, 2012.

2. P. Zarka, et al., "NenuFAR: Instrument Description and Science Case", International Conference on Antenna Theory and Techniques (ICATT), Kharkiv, Ukraine, pp. 13-18, April, 2015.

3. The NenuFAR-France consortium ( $~ 80$ co-authors), "NenuFAR : instrument description and science case", June 2014, latest version on $\underline{h t t p: / / n e n u f a r . o b s-n a n c a y . f r / I M G / p d f / n e n u f a r-s c i e n c e-c a s e-v 5 \_2014 \_10 \_10 \_p z . p d f}$ 